\title{
NOTE ON THE CURVATURE OF ORTHOGONAL TRAJECTORIES OF LEVEL CURVES OF GREEN'S FUNCTION. III
}

\author{
J. L. WALSH
}

If $R$ is a simply connected region of the extended $(x, y)$-plane with boundary $B$, and if Green's function $G(x, y)$ exists for $R$ with pole in the finite point $O$, we denote by $\{T\}$ the set of orthogonal trajectories to the level curves $G(x, y)=\log r, 0<r<1$, in $R$. The totality of circles each osculating at $O$ one of the set of curves $T$ passing through $O$ consists precisely of the set of circles through $O$ and through another fixed point $D$, depending on $O$ and $R$. The point $D$ is called the conjugate of $O$ with respect to $R$. The term "circle" is here and below used in the extended sense, to include straight line, unless otherwise noted.

In a series of papers ${ }^{1}$ the writer has recently studied some of the properties of the point $D$, notably (in $\mathrm{M}$ and I) that every circle through $O$ and $D$ cuts $B$; and (in II) that every point exterior to $R$ is the conjugate with respect to $R$ of a suitably chosen point $O$ interior to $R$. It is the object of the present note to establish still further properties of the conjugate, namely the following theorems:

THEOREM 1. Let $R$ be a simply connected region of the w-plane with at least two boundary points. Let $C$ be a circle intersecting the boundary of $R$ in the finite point $\alpha$. Let $C$ be the boundary of a circular region $R^{\prime}$ (a half-plane, interior of a circle, or exterior of a circle, boundary points not included) whose points lie in $R$, and let $T$ be a triangle contained in $R^{\prime}$, with the vertex $\alpha$. Let the sequence of points $w_{1}, w_{2}, \cdots$ lie in $T$ and approach $\alpha$. Then the conjugate of $w_{n}$ with respect to $R$ also approaches $\alpha$ as $n$ becomes infinite.

THEOREM 2. Let $R$ be a simply connected region of the w-plane with at least two boundary points, and let $w_{0}$ be a boundary point of $R$. Then there exists a sequence of points $w_{1}, w_{2}, \cdots$ in $R$ approaching $w_{0}$ such that the conjugate of $w_{n}$ with respect to $R$ approaches $w_{0}$.

THEOREM 3. There exists a limited Jordan region $R$ of the w-plane, a boundary point $w_{0}$ of $R$, and a sequence $w_{1}, w_{2}, \cdots$ of points of $R$ approaching $w_{0}$ such that the conjugate of $w_{n}$ with respect to $R$ becomes infinite with $n$.

${ }^{1}$ American Mathematical Monthly, vol. 42 (1935), pp. 1-17; Proceedings of the National Academy of Sciences, vol. 23 (1937), pp. 166-169; this Bulletin, vol. 44 (1938), pp. 520-523. We shall refer to these papers as M, I, II respectively. 
Let $w=f(z)$ map $|z|<1$ onto $R$. Then the conjugate of $w$ with respect to $R$ is the point

$$
w^{\prime}=f(z)-\frac{2\left[f^{\prime}(z)\right]^{2}(1-z \bar{z})}{f^{\prime \prime}(z)(1-z \bar{z})-2 \bar{z} f^{\prime}(z)} ;
$$

this is the function whose continuity (as a function of $w$ ) we are studying.

1. Proof of Lemma 1. As a step in the proof of Theorem 1 we establish a preliminary result.

Lemma 1. Let the finite point $O$ lie interior to the simply connected region $R$ whose Green's function with respect to $O$ exists, and let each point of the boundary $B$ of $R$ lie on or to the right of the vertical line $O y$. Let at least one point $A$ of $B$ lie to the right of $O y$. Then the conjugate $D$ of $O$ with respect to $R$ is finite and lies to the right ${ }^{2}$ of $O y$.

In the proof it is convenient to transform $O$ to infinity by a linear transformation of the complex variable, so the boundary $B$ of $R$ can now be supposed finite. We preserve the original notation. The conjugate of $O$ is a finite point $D$, the conformal center of gravity ${ }^{3}$ of $R$. There exists a line $L$ with (for suitable orientation of the plane) each point of the boundary $B$ of $R$ lying on or to the right of $L$, and with at least one point $A$ of $B$ lying to the right of $L$. For definiteness let $A$ be the point (or one of the points) of $B$ farthest from $L$.

If no boundary point of $R$ lies on $L$, a suitably chosen level curve of Green's function $G(x, y)$ for $R$ with pole in $O$ lies to the right of $L$. The center of gravity of this level curve with positive mass distribution defined by the equation

$$
d \sigma=\frac{\partial G}{\partial n} d s
$$

is the conjugate of $O$ with respect to $R$, and lies to the right of $L$. The conclusion of Lemma 1 follows in this case. Henceforth we suppose at least one point of $B$ to lie on $L$.

An arbitrary cut in the region $R$ (assumed to lie in the w-plane) corresponds under the conformal $\operatorname{map} \zeta=\zeta(w)$ of $R$ onto $|\zeta|>1$ to a cut in the region ${ }^{4}|\zeta|>1$. Let a circle $K$ be drawn with center $A$ and radius one-half the distance from $A$ to $L$. A certain simply connected

${ }^{2}$ It follows (see II) that $O$ cannot be a maximum or even a critical point of the function $r(a)$.

${ }^{3}$ See $M$ and $I$.

${ }^{4}$ See for instance Carathéodory, Conformal Representation (Cambridge Tract, no. 28$)$, p. 83 . 
region $S$ consisting of points of $R$ and containing some points of $R$ lying to the right of $A$, lies interior to $K$ and is bounded entirely by points of $B$ and of $K$. That is to say, we define $S$ as the set of points $P$ each of which can be joined to a fixed point $H$ in $K$ and $R$ to the right of $A$, by a Jordan arc $P H$ not cutting $B$ or $K$. The boundary of $S$ contains at least one arc $Q$ of $K$ whose end-points are points of $B$ but which otherwise lies in $R$; all such $\operatorname{arcs} Q$ (by hypothesis belonging to the boundary of $S$ ) are finite or denumerably infinite in number. Under the conformal map each arc $Q$ corresponds to a Jordan arc $Q_{1}$ in the region $|\zeta|>1$ whose end-points are distinct points of $|\zeta|=1$, whether or not the two end-points of $Q$ are distinct. ${ }^{5}$ Under the conformal map the region $S$ corresponds to a region $S_{1}$ bounded in part by $\operatorname{arcs} Q_{1}$. We now prove that the boundary of $S_{1}$ must contain at least one arc of the circle $|\zeta|=1$.

Two distinct $\operatorname{arcs} Q_{1}$ may have a terminal point $T_{1}$ in common, but if so the corresponding arcs $Q$ have a terminal point $T$ in common, and a suitably chosen neighborhood of $T$ contains no boundary points of $S$ interior to $K$. For there exists a sequence of Jordan arcs $J_{1}$ in $S_{1}$ with their end-points on the respective $\operatorname{arcs} Q_{1}$; and the end-points of the $J_{1}$ approach $T_{1}$. Let a point $M_{1}$ of $S_{1}$, whose transform is the point $M$ of $S$, be separated by each of these Jordan arcs plus the boundary of $S_{1}$ from the neighborhood of $T_{1}$ in $S_{1}$. The corresponding sequence of Jordan $\operatorname{arcs} J$ in $S$ have their end-points on the respective $\operatorname{arcs} Q$, and each arc $J$ together with the boundary of $S$ separates $M$ from the neighborhood of at least one boundary point of $S$. If each of the Jordan arcs $J$ together with the boundary of $S$ separates a neighborhood in $S$ of more than one boundary point from $M$, let $E$ denote the totality of such boundary points. No point of $E$ not an end-point of an $\operatorname{arc} Q$ can be a limit point of a sequence of boundary points of $S$ not lying on $E$; for two nonintersecting arcs $J$ together with the two subarcs of arcs $Q$ intercepted between their terminal points bound a Jordan region in $S$ which contains then no point of the boundary of $S$ in its interior; of course $S$ is simply connected, and no boundary point of $S$ lies exterior to $K$. If $E$ contains more than one point, it contains either a point interior to $K$ or a point on $K$ not an end-point of $\operatorname{arcs} Q$ (hence lying on an arc of $K$ belonging to $B$ ), and in any case contains a point $N$ with the property that the mapping function $\zeta=\zeta(w)$ for $R$ is continuous in some neighborhood of $N$ in $S$ and constant (equal to the value of $\zeta$ in the point $T_{1}$ ) at every boundary point of $S$ in the neighborhood, which is impossible. ${ }^{6}$ It follows that $E$ consists of a

${ }^{5}$ Carathéodory, op. cit., p. 85.

${ }^{6}$ Carathéodory, op. cit., p. 82. 
single point $T$; a suitably chosen neighborhood of $T$ contains no boundary point of $S$ interior to $K$.

The method of proof just given shows also that a point $T_{1}$ of $|\zeta|=1$, not an end-point of an arc of $|\zeta|=1$ which is part of the boundary of $S_{1}$ but which is a limit point of an infinity of Jordan arcs $Q_{1}$, corresponds in the sense just considered to a single point $T$ on $B$ and $K$; a suitably chosen neighborhood of $T$ contains no boundary point of $S$ interior to $K$. The point $A$ is not an interior point of $S$, so it follows that there exists some point of $K$ which is a limit point of boundary points of $S$ interior to $K$, whence not all points of $B$ on $K$ can be points $T$ as considered above, and the boundary of $S_{1}$ must contain at least one arc of the circle $|\zeta|=1$, say of length $\sigma>0$, as we desired to prove.

Denote by $d$ the distance from $A$ to $L$. Choose $r,(0<r<1)$, so near unity that an arc of the Jordan curve $B_{r}: G(x, y)=\log r$ interior to $K$ and $S$ corresponds to an arc of $|\zeta|=1 / r$ in $S_{1}$ of length greater than $\sigma / 2 r$, which is possible by inspection of the situation in the $\zeta$-plane; and also choose $r$ so near to unity that no point of the curve $B_{r}$ lies at a greater distance from $B$ than some positive number

$$
d^{\prime}<\sigma d /(8 \pi-2 \sigma) \text {. }
$$

Then no point of the curve $B_{r}$ lies at a distance greater than $d^{\prime}$ to the left of $L$. The weight of that part of $B_{r}$ interior to $K$ is at least $\sigma / 2$. The center of gravity of the weighted locus $B_{r}$ lies to the right of $L$ at a distance not less than

$$
\left\{\frac{\sigma}{2} \frac{d}{2}-\left(2 \pi-\frac{\sigma}{2}\right) d^{\prime}\right\} / 2 \pi,
$$

which is positive. That is to say, the center of gravity of the weighted locus $B_{r}$ (that is, the conformal center of gravity of $R$, or the conjugate of $O$ with respect to $R$ ) lies to the right of $L$; so Lemma 1 follows.

2. Proof of Theorem 1. For convenience suppose the plane oriented so that $\alpha$ lies at the right-hand extremity of a horizontal diameter of $C$. Let $R_{n}$ be the region of the w-plane obtained from $R$ by stretching without rotation so that final and initial lengths are in the ratio $1: r\left(w_{n}\right)$, with the new transform of $w_{n}$ corresponding to the origin. Here (as in II) we use $r\left(w_{n}\right)$ to denote the inner radius of $R$ with respect to the point $w_{n}$. From each sequence $R_{n}$ can be extracted a subsequence of regions converging to a kernel in the sense of Carathéodory. For the corresponding functions $w=f_{n}(z)$ which map $|z|<1$ onto $R_{n}$ are univalent with $f_{n}(0)=0$ and $\left|f_{n}^{\prime}(0)\right|=1$, hence 
form a normal family in $|z|<1$. Let us suppose a subsequence of the $R_{n}$ to converge to the kernel $R_{0}$, with the corresponding mapping functions $w=f_{n}(z)$ converging continuously in $|z|<1$ to the mapping function $w=f_{0}(z)$ for $R_{0}$, with $f_{0}(0)=0,\left|f_{0}^{\prime}(0)\right|=1$. The kernel $R_{0}$ is obviously not a single point. Then we have for the subsequence

$$
\lim _{n \rightarrow \infty}-2 \frac{\left[f_{n}(0)\right]^{2}}{f_{n}^{\prime \prime}(0)}=-2 \frac{\left[f_{0}^{\prime}(0)\right]^{2}}{f_{0}^{\prime \prime}(0)}
$$

or in other words (compare I) the conjugate of the origin with respect to $R_{n}$ approaches the conjugate of the origin with respect to $R_{\mathbf{0}}$.

We shall henceforth assume that the sequence $R_{n}$ itself converges to $R_{0}$ and that (1) is valid. This assumption involves no loss of generality, for if the conclusion of the theorem is false it is false for a sequence of indices such that $R_{n}$ converges to a kernel and such that (1) is valid for that sequence.

We shall now assume that the angle (argument, amplitude) $\angle\left(\alpha-w_{n}\right)$ approaches a limit $\gamma$ as $n$ becomes infinite; this assumption likewise involves no loss of generality, for if the theorem is false there exists a subsequence of the $w_{n}$ for which this angle approaches a limit and whose conjugates do not approach $\alpha$. Of course $\gamma$ is not the angle $\pi / 2$ or $-\pi / 2$.

Denote by $t_{n}$ the boundary point of $R$ nearest to $w_{n}$; then $t_{n}$ lies on or exterior to $C$ :

$$
\left|w_{n}-t_{n}\right| \geqq a-\left|w_{n}\right|,
$$

where $C$ is the circle $|w|=a$; here we assume also that $C$ is a proper circle in whose interior $T$ lies. This assumption involves no loss of generality. The inequality

$$
\left|w_{n}-t_{n}\right| \leqq r\left(w_{n}\right)
$$

is well known. ${ }^{7}$ Moreover for suitably chosen $\delta>0$ independent of $n$ we have

$$
\delta\left|w_{n}-\alpha\right| \leqq a-\left|w_{n}\right|
$$

by virtue of the fact that $w_{n}$ lies in the triangle $T$. We obviously have, by (2), (3), and (4),

$$
\left|w_{n}-\alpha\right| / r\left(w_{n}\right) \leqq 1 / \delta
$$

There is no boundary point of $R_{n}$ or of $R_{0}$ closer to $O$ than the dis-

${ }^{7}$ See for instance P6lya-Szegö, Aufgaben und Lehrsätze aus der Analysis, vol. 2, Berlin, 1925, p. 21, exercise 121. 
tance $1 / 4$, by the distortion theorem (Verzerrungssatz). But by the inequality just established there is a boundary point of $R_{n}$ (namely the transform of the point $\alpha$ ) on the line segment from $O$ in the direction $\angle\left(\alpha-w_{n}\right)$ of length $1 / \delta$, so there is a boundary point of the kernel $R_{0}$ on the line segment from $O$ in the direction $\gamma$ of length $1 / \delta$. Consequently $R_{0}$ has at least one boundary point which lies to the right of the vertical line through $O$.

It follows from Lemma 1 that the conjugate of $O$ with respect to $R_{0}$ is not infinite, follows from (1) that the conjugate of $O$ with respect to $R_{n}$ approaches a finite limit, and follows from the relation $r\left(w_{n}\right) \rightarrow 0$ (proved in II) that the distance from $w_{n}$ to its conjugate with respect to $R$ (which is $r\left(w_{n}\right)$ multiplied by the distance from $O$ to its conjugate with respect to $R_{n}$ ) approaches zero. Theorem 1 is established.

It is clear from the proof of Theorem 1 that the essential part of the proof is that every kernel $R_{0}$ of a subsequence of the regions $R_{n}$ should have the property that the conjugate of the origin with respect to $R_{0}$ shall be finite; whenever the sequence $R_{n}$ has this property, the condition $w_{n} \rightarrow \alpha$ implies that the conjugate of $w_{n}$ with respect to $R$ also approaches $\alpha$.

3. Proof of Theorem 2. Thanks to Theorem 1, the proof of Theorem 2 is extremely simple. For definiteness suppose $w_{0}$ finite (the contrary case can be reduced to this by a linear transformation). Let $\omega_{k}$ be a point of $R$ whose distance from $w_{0}$ is less than $1 / 2^{k}$. Let $\gamma_{k}$ be the circle whose center is $\omega_{k}$ and whose radius is the distance from $\omega_{k}$ to the boundary of $R$; this distance is necessarily less than $1 / 2^{k}$. The interior of this circle lies in $R$, but at least one point $\alpha$ of the circumference is a boundary point of $R$. A triangle $T$ satisfying the requirements of Theorem 1 can be constructed. By Theorem 1 there exists a point $w_{k}$ interior to $\gamma_{k}$ whose conjugate with respect to $R$ lies in the circle $\left|w-w_{0}\right|=1 / 2^{k-1}$, and we have $\left|w_{k}-w_{0}\right| \leqq 1 / 2^{k-1}$. The sequence $w_{k}$ satisfies the requirements of Theorem 2 .

4. Proof of Theorem 3. The region $R$ whose existence is asserted in Theorem 3 is now to be constructed by the following method. ${ }^{8} \mathrm{We}$ consider the sequence of circles $C_{n}$ in the $w(=x+i y)$-plane, each tangent to its predecessor :

$$
\begin{gathered}
C_{0}: \quad x^{2}+y^{2}=1, \quad C_{1}: \quad(x-3 / 2)^{2}+y^{2}=1 / 4, \\
C_{n}: \quad\left(x-3\left(2^{n}-1\right) / 2^{n}\right)^{2}+y^{2}=1 / 2^{2 n} .
\end{gathered}
$$

8 This method is quite similar to one employed for a somewhat different purpose in a forthcoming paper by Seidel and Walsh, of which an abstract was published in Proceedings of the National Academy of Sciences, vol. 24 (1938), pp. 337-340. 
The interiors of these circles are to be joined by canals in the neighborhoods of the points

$$
1,2,5 / 2, \cdots, \alpha_{n}: 3-1 / 2^{n-1}, \cdots
$$

so as to form a Jordan region; the banks of the canals are short segments of lines parallel to the axes of reals, a short distance above and below that axis near the points $\alpha_{n}$ of tangency of successive circles; the arcs of the original circles intercepted between those lines are to be suppressed, and the banks of the canals are to be terminated by successive circumferences. We proceed to indicate in more detail the construction of the canals.

Let the canal in the neighborhood of the point $\alpha_{n}$ be bounded by the lines $y= \pm \delta_{n}$, with $\delta_{n}>0$. Let $R$ be the region formed by the interior of the circles $C_{n}$ together with the canals, and let the point $w=0$ correspond to the point $\zeta=0$ when $R$ is mapped onto $|\zeta|<1$ with directions at the origins unaltered. When $\delta_{1}$ approaches zero, the kernel of the variable region $R$ in the sense of Carathéodory (op. cit., p. 75) is precisely the interior of $C_{0}$, independently of the values of $\delta_{2}, \delta_{3}, \cdots$. The function $w=f_{0}(\zeta)$ which maps $|\zeta|<1$ onto $R$ with $f_{0}(0)=0, f_{0}^{\prime}(0)>0$, approaches the function $w=\phi_{0}(\zeta) \equiv \zeta$ which maps $|\zeta|<1$ onto $R$ with $\phi_{0}(0)=0, \phi_{0}^{\prime}(0)>0$; convergence is uniform in every $|\zeta| \leqq r<1$. The conjugate of the origin with respect to $R$ is $-2\left[f_{0}^{\prime}(0)\right]^{2} / f_{0}^{\prime \prime}(0)$, which approaches the conjugate of the origin with respect to the interior of $C_{0}$, namely the point at infinity. Consequently it is possible to choose $\delta_{1}$ so small independently of $\delta_{2}, \delta_{3}, \ldots$ that the conjugate of the point $w_{0}=0$ with respect to $R$ lies exterior to the circle $|w|=1$.

Introduce the notation for the center of $C_{n}: w_{n}=3\left(2^{n}-1\right) / 2^{n}$. When $\delta_{1}$ and $\delta_{2}$ approach zero, the kernel of the variable region $R$ is the interior of $C_{1}$ independently of $\delta_{3}, \delta_{4}, \ldots$, if the region $|\zeta|<1$ is mapped onto $R$ by the function $w=f_{1}(\zeta)$ with $f_{1}(0)=w_{1}$, $f_{1}^{\prime}(0)>0$. Then the function $w=f_{1}(\zeta)$ approaches the function $w=\phi_{1}(\zeta) \equiv w_{1}+\zeta / 2$ which maps $|\zeta|<1$ onto the interior of $C_{2}$ with $\phi_{1}(0)=w_{1}, \phi_{1}^{\prime}(0)>0$; convergence is uniform in every $|\zeta| \leqq r<1$. The conjugate of $w_{1}$ with respect to $R$ is $-2\left[f_{1}^{\prime}(0)\right]^{2} / f_{1}^{\prime \prime}(0)$, which approaches the conjugate of $w_{1}$ with respect to the interior of $C_{1}$, namely the point at infinity. Consequently it is possible to choose $\delta_{1}$ and $\delta_{2}$ so small independently of $\delta_{3}, \delta_{4}, \cdots$ that the conjugate of the point $w_{1}$ with respect to $R$ lies exterior to the circle $|w|=2$. The number $\delta_{1}$ has been subjected to a similar restriction in connection with the conjugate of the point $w_{0}$, and is to be subjected to no further condition.

We continue now in the way thus commenced. The numbers $\delta_{n}$ and 
$\delta_{n+1}$ are to be chosen independently of $\delta_{n+2}, \delta_{n+3}, \cdots$ in such manner that the conjugate of the point $w_{n}$ with respect to $R$ lies exterior to the circle $|w|=2^{n+1}$; each $\delta_{n}$ (for $n>1$ ) is subjected then to two conditions, and the numbers $\delta_{n}$ can be determined in succession. The resulting region $R$ is a Jordan region. The sequence $w_{n}$ approaches the boundary point $w=3$ of $R$, and the conjugate of $w_{n}$ with respect to $R$ becomes infinite with $n$. Theorem 3 is established.

HARVARD UNIVERSITY

\section{ON THE ORDER OF THE PARTIAL SUMS OF FOURIER POWER SERIES ${ }^{1}$}

\section{OTTO SZÁsZ}

Dedicated to L. Fejér on his sixtieth birthday.

Let $f(x)$ be a Lebesgue integrable function, and denote the partial sums of its Fourier series by $s_{n}(f ; x)$. It is well known that $s_{n}=o(n)$ uniformly ${ }^{2}$ in $x$. Recently W. C. Randels ${ }^{3}$ gave an example showing that this estimate cannot be improved. The same conclusion can be drawn from a note by E. C. Titchmarsh ; ${ }^{4}$ and A. Zygmund in his review of Randels' article (Zentralblatt für Mathematik, vol. 18, p. 353) pointed to another device, using convex coefficient sequences, which would establish the same fact.

In this note a simple construction is given, using a sequence of polynomials in the complex variable $z$. This leads to a sharper result showing that even for Fourier power series (that is, a power series considered on its circle of convergence and integrable) the estimate cannot be improved. Moreover, an example $F(z)=\sum_{n=0}^{\infty} c_{n} z^{n}$ is given which has the additional property that $F(z) /(1-z)$ is a generalized Fourier power series on $|z|=1$.

We start with a sequence of polynomials of increasing degree $P_{n}(z)=\left(\sum_{v=0}^{m} c_{n v} z^{\nu}\right)^{2}=\sum_{v=0}^{2 m} a_{n v} z^{\nu}$ having the following properties:

$$
\frac{1}{2 \pi} \int_{-\pi}^{\pi}\left|P_{n}\left(e^{i x}\right)\right| d x=\sum_{\nu=0}^{m}\left|c_{n \nu}\right|^{2}=1
$$

1 Presented to the Society, April 15, 1939.

${ }^{2}$ In fact, if $c_{0}, c_{1}, \cdots$ are the Fourier coefficients, then $c_{n} \rightarrow 0$. Hence $\sum_{0}^{n}\left|c_{\nu}\right|=o(n)$.

${ }^{3} \mathrm{~W}$. C. Randels, On the order of the partial sums of a Fourier series, this Bulletin, vol. 44 (1938), pp. 286-288.

${ }^{4}$ E. C. Titchmarsh, Principal value Fourier series, Proceedings of the London Mathematical Society, (2), vol. 23 (1925), pp. xli-xliii. 